\title{
Intracranial hemangioblastomas: An institutional experience
}

\author{
Srinivas Dwarakanath, Ashish Suri, Bhavani Shanker Sharma, Veer Singh Mehta \\ Department of Neurosurgery, Neurosciences Center, All India Institute of Medical Sciences, New Delhi - 110029, India
}

Background and Aims: We present our Institutional experience with intracranial hemangioblastomas. Settings and Design: A retrospective study. Materials and Methods: This study included all patients of intracranial hemangioblastomas admitted in our institution over a period of 11 years from January1992 through June 2003. Results: There were a total of 69 patients ( 45 males and 24 females). The average age at presentation was 34.5 years. The tumor was located in the cerebellar hemispheres, vermian and brainstem regions in $42(60 \%)$ patients, $19(28 \%)$ patients and $8(12 \%)$ patients, respectively. Hydrocephalus was seen in $48(69 \%)$ patients. Thirty-three patients underwent CSF diversion procedures prior to surgery on the tumor. All except one underwent definitive surgery. The mortality was $8(11 \%)$. Sixty eight patients underwent surgery on the tumor. The follow-up ranged from 1 month to 11 years. Fifteen patients developed recurrent lesions. Conclusion: Lifelong surveillance is necessary in cases with hemangioblastomas to identify recurrences especially in those patients having VHL syndrome.

Key words: Hemangioblastomas, Intracranial

\section{Introduction}

Cushing and Bailey coined the term 'Hemangioblastomas' to describe tumors arising from the endothelial cells of the central nervous system. ${ }^{[1]}$ Though histologically benign, their development is often unfavorable due to high frequency of recurrence and multicentricity especially when occurring in a familial set-up i.e., Von Hippel Lindau (VHL) syndrome. ${ }^{[2]}$ They account for 1.5$2.5 \%$ of all intracranial and 7-12\% of posterior fossa tumors. Surgery is the standard modality of treatment though their hypervascularity and location present a formidable challenge. ${ }^{[1]}$ In this study we have analysed our experience with hemangioblastomas and reviewed in detail the available literature. ${ }^{[3-12]}$

\section{Materials and Methods}

This retrospective study included all patients of neuropathologically verified hemangioblastomas treated over a period of 11 years from January1992 through December 2003.

\section{Results}

There were a total of 69 patients with cranial hemangioblastomas. Among them 45 were males and 24 females (ratio of 1.9:1). The ages ranged between 12 to 70 years with the average being 34.4 years. Twenty-six patients were in the third decade (38\%) and 14 were in the fourth decade of life. The duration of symptoms prior to admission ranged from 2 days to 12 years with an average of 7.5 months. The clinical features are elaborated in the Table 1. Headache was the commonest symptom occurring in $61(88 \%)$ patients followed by symptoms suggestive of cerebellar involvement in $55(80 \%)$ patients. Forty nine $(71 \%)$ had associated recurrent vomiting, while $21(30 \%)$ patients presented with visual deterioration. The average duration of visual deterioration was

\begin{tabular}{lcc}
\hline \multicolumn{2}{c}{ Table 1: Clinical features } \\
\hline Symptoms & Number of patients & Percentage \\
Headache & 61 & 88 \\
Cerebellar ataxia & 55 & 80 \\
Vomiting & 49 & 71 \\
Visual deterioration & 21 & 30 \\
Cranial n involvement & 6 & 8 \\
Motor & 2 & 3 \\
Sensory & 1 & 1.6 \\
Signs & & \\
Papilloedema & 44 & 63 \\
Sec optic atrophy & 6 & 8 \\
Cranial N involvement & & \\
$\quad$ IIIIV & 1 & 1.4 \\
$\quad$ V & 1 & 1.4 \\
$\quad$ VI & 6 & 9 \\
VII & 8 & 13 \\
$\quad$ VIII & 3 & 4 \\
$\quad$ IXIX & 13 & 19 \\
Motor & 8 & 11.5 \\
Sensory & 1 & 1.4 \\
Cerebellar & 63 & 91 \\
\hline
\end{tabular}


4.5 months. Six patients were diagnosed with VHL syndrome. Of these, 2 were diagnosed preoperatively while 4 were diagnosed to have VHL syndrome following surgery.

The tumor was located in the cerebellar hemispheres, vermian and brainstem regions in $42(60 \%)$ patients, 19 (28\%) patients and $8(12 \%)$ patients, respectively. Tumors in $51(73 \%)$ patients were predominantly cystic while in $18(27 \%)$ patients these were predominantly solid or with a small cystic component. Varying degrees of hydrocephalus was present in 48 (69\%) patients.

Thirty-three patients underwent a CSF diversion procedure prior to surgery on the tumor. Sixty-eight patients underwent a definitive surgery for excision of the tumor while 1 patient was treated primarily with Gamma knife and did not undergo surgery.

Total excision was done in 63 of the 68 patients. Relationship with brainstem and cranial nerves limited the totality of tumor resection in rest of the five cases. Eight (11\%) patients died in the post-operative period. In five, septicemia was the predominant cause and all these had a turbulent and prolonged postoperative course. Two patients had massive air embolism intraoperatively and died in the early postoperative period. Both of these patients were operated in the sitting posture. One patient had a massive myocardial infarction. Fifteen (17\%) developed postoperative complications. Of these 11 patients developed complications after the primary surgery while 4 patients developed complications after surgery for recurrent tumors. Chest infection, deterioration in cranial nerve function, CSF leak and meningitis were the complications observed in 4 patients each. One patient each developed drug-induced nephropathy, deterioration in motor power, postural hypotension and urinary tract infection. All these patients gradually improved.

Fifty-three $(87 \%)$ patients were followed up for a duration ranging from one month to 11 years. Among these 53 patients, 31 patients have been followed up for 3 years or more. 15 patients have a follow-up of 1-3 years. The rest have a follow-up of less than one year. Eight patients were lost to follow-up. Eleven patients required a VP shunt insertion on follow-up while 1 patient had his shunt removed due to shunt infection. Fifteen (21\%) patients developed recurrences, of which 10 patients had a single recurrence while 5 patients had 2 recurrences. Of the patients, who had recurrences, 11 patients developed the recurrence at the same site of the previous tumor while 4 developed a recurrence at another site (opposite cerebellar hemisphere). The average time taken for the primary recurrence was 4.5 years and 4.6 years for the secondary recurrence.

Two patients underwent Gamma knife therapy. One underwent GK as a primary and only procedure while one patient was given GK after he developed a recurrence. One of these two patients was lost to follow up while the other patient had an increase in the cystic component, which required surgical evacuation.

\section{Discussion}

Hemangioblastomas may occur sporadically or as part of VHL complex. ${ }^{[3]}$ In this study, the male/female ratio is closer to the observation of Boughey et.al but higher compared to the other series. ${ }^{[3,4-13]}$ The average age at presentation was 34.5 -years, which is less than the reports in literature where it has ranged from 3849.5 years. ${ }^{[4-12]}$ VHL syndrome was seen in $9 \%$ (6 patients), which is less than the $23-40 \%$ recorded in literature. ${ }^{[5]}$

The triad of headache, vomiting and cerebellar symptoms has been the most common clinical features in literature. ${ }^{[4-12]}$ Fifty patients in our series had features of raised intracranial pressure. This is similar to the previous series in which the incidence of papilloedema has ranged from 39-70\% and cerebellar involvement from $40-80 \%$. Cranial nerve involvement seen in $14(20 \%)$ of our patients has varied from $17-35 \%$ in literature. ${ }^{[4-12]}$

There may be erythrocytosis and is believed to be due to secretion of Erythropoietin-like substance by the neoplastic tissue (30). The average hemoglobin in our study was $13.5 \mathrm{~g} \%$ compared to an average of $16-18 \mathrm{~g} \%$ reported in literature. ${ }^{[9,12]}$

Radiologically, 51 were predominantly cystic while 18 were either solid or predominantly solid with a small cystic component, which corresponds to figures in available literature (60-72\% are cystic tumors). ${ }^{[5-10]}$ In the present series, tumors in the cerebellar hemispheres accounted for 41(60\%), vermian tumors in 19 (28\%) while brainstem (subpial) tumors were seen in $9(12 \%)$ patients, which is similar to available literature. Hydrocephalus seen in 48 (69\%) patients was significantly higher than the $20 \%$ mentioned in literature. ${ }^{[9]}$ This could be in part due to the delayed presentation/ referral of our patients.

Complete excision is the goal of the surgery. The cyst wall need not be removed. The solid nodule tumor mass is removed microscopically by dissecting the gliotic margins away from the tiny vascular pedicles that are coagulated. To avoid intraoperative swelling, the large draining veins have to be preserved until the arterial feeders to the mural nodule have been isolated and resected. Piecemeal removal must be avoided to prevent catastrophic bleeding. Some authors have advocated the use of preoperative embolisation though none of our patients underwent the same.

The mortality rate in available literature has varied from 4$36 \% .^{[3,5-9,11,12,14-16]}$ The mortality in this series was $11 \%$ (8 patients). In the present series 15 patients (17\%) developed postoperative complications which corresponds to available literature. ${ }^{[3,5-9,11,12,15-16]}$ The common postoperative morbidity was due to chest infection, lower cranial nerve paresis and meningitis.

Factors predicting a poor outcome are multiple hemangioblastomas, association with retinal hemangioblastomas and/or onset of disease at less than 30 years age. ${ }^{[5]}$ In this study predominantly solid and midline tumors had a significantly higher mortality when compared to cystic and hemispheric tumors ( $P$ $<0.05$ ), which can be partly explained by their proximity to the brainstem. $^{[7,8,12]}$

Dela Monte stated that recurrence was correlated with younger age ( < 30 years) and VHL syndrome. ${ }^{[17]}$ Also, cystic tumors were more frequent, had a longer survival while recurrence was less. ${ }^{[6]}$ While cystic tumors were more common in this study we could not find a correlation between them and the age at 
presentation and recurrence. In literature, recurrence has ranged from $16-30 \% .^{[3,5,7,12,15-17]}$ In the present series 15 (21\%) patients developed recurrences. There was no change in the histological features in these recurrences.

Pan et al treated 20 haemangioblastomas with Gamma-Knife (GK). ${ }^{[18]}$ Niemala et al treated 10 patients with 11 hemangioblastomas with GK. ${ }^{[19]}$ They suggested that a solitary small or medium-sized hemangioblastomas usually shrink or stop growing after radiosurgery.

\section{References}

1. Rengachary SS, Blount JP. Hemangioblastomas. In Wilkins RH, Rengachary SS, editors. Neurosurgery, $2^{\text {nd }}$ ed. McGraw Hill: 1996. p. 1205-19.

2. Melmon KL, Rosen SW. Lindau's disease: Review of the literature and study of large kindred. Am J Med 1964;36:565-617.

3. Boughey AM, Fletcher NA, Harding AE. Central nervous system hemangioblastomas: A clinical and genetic study of 52 cases. J Neurol Neurosurg Psychiatr 1990;54:644-8.

4. Conway JE, Chou D, Clatterbuck RE, Brem H, Long DM, Rigamonti D Hemangioblastomas of the central nervous system in von Hippel-Lindau syndrome and sporadic disease. Neurosurgery 2001;48:55-63.

5. Resche F. Infratentorial hemangioblastomas [Abstract in English]. Neurochirurgie 1985;31:91-149.

6. Julow J, Balint K, Gortvai P, Pasztor E. Posterior fossa hemangioblastomas. Acta Neurochir (Wien) 1994;128:109-14.

7. Symon L, Murota T, Pell M, Bordi L. Surgical management of haemangioblastoma of the posterior fossa. Acta Neurochir (Wien) 1993;120:103-10.

8. Palmer H. Hemangioblastomas. A review of 81 cases. Acta Neurochir (Wien) $1972 ; 27: 125-48$

9. Jeffreys R. Clinical and surgical aspects of posterior fossa hemangioblastomata. J Neurol Neurosurg Psychiatr 1975;38:105-11.

10. Boughey AM. Fletcher NA, Harding AE. Central nervous system hemangioblastomas: A clinical and genetic study of 52 cases. J Neurol Neurosurg Psychiatr 1990;54:644-8.

11. Obrador S, Martin-Rodriguez JG. Biological factors involved in the clinical features and surgical management of cerebellar hemangioblastomas. Surg Neurol $1977 ; 7(2): 79-85$.

12. Constans JP, Meder F, Maiuri F, Donzelli R, Spaziante R, de Divitiis E. Posterior fossa hemangioblastomas. Surg Neurol 1986;25:269-75.

13. Elster AD, Arthur DW. Intracranial hemangioblastomas: CT and MR findings. J Comput Assist Tomogr 1988;12:736-9.

14. Weil RJ, Lonser RR, DeVroom HL, Wanebo JE, Oldfield EH. Surgical management of brainstem hemangioblastomas in patients with von Hippel-Lindau disease. J Neurosurg 2003;98:95-105.

15. Mondkar VP, McKissock W, Russell RW. Cerebellar haemangioblastomas. Br J Surg 1967;54:45-9.

16. Londrini S, Lasio G, Cimino C, Pluchino F. Hemangioblastomas: Clinical characteristics, surgical results and immunohistochemical studies. J Neurosurg Sci 1991;35:179-85.

17. de la Monte SM, Horowitz SA. Hemangioblastomas: Clinical and histopathological factors correlated with recurrence. Neurosurgery 1989;25:695-8.

18. Pan L, Wang EM, Wang B.J, Zhou LF, Zhang N, Cai PW, et al. Gamma knife radiosurgery for hemangioblastomas. Stereotact Funct Neurosurg 1998;70:179-86.

19. Niemela M, Lim Y.J, Soderman M, Jaaskelainen J, Lindquist C. Gamma knife radiosurgery in 11 hemangioblastomas. J Neurosurg 1996;85:591-6. 